\title{
Giant increase in cross-magnetic-field transport rate as an electron-positron plasma cools
}

\author{
F. F. Aguirre and C. A. Ordonez ${ }^{\text {a) }}$ \\ Department of Physics, University of North Texas, Denton, Texas 76203, USA
}

(Received 25 August 2017; accepted 21 September 2017; published online 5 October 2017)

\begin{abstract}
An electron-positron plasma in thermal equilibrium within a uniform magnetic field is studied using a classical trajectory Monte Carlo simulation. The cross-magnetic-field single-particle diffusion coefficient is evaluated as a function of the magnetic field strength and plasma temperature. The transport rate is found to increase by many orders of magnitude as the plasma temperature is lowered, for a magnetic field strength of $1 \mathrm{~T}$. The sharp dependence on temperature is due to electrons and positrons becoming temporarily correlated and drifting across the magnetic field before dissociating. Published by AIP Publishing. https://doi.org/10.1063/1.5001680
\end{abstract}

The quest for high precision measurements of the spectral and gravitational properties of antimatter has led to a search for new ways to increase the yield in the production of cold antihydrogen. ${ }^{1-4}$ There exists renewed interest in producing antihydrogen using a reaction involving an excited positronium atom with an antiproton because the cross section for the formation of antihydrogen increases dramatically at higher positronium-atom energy levels. ${ }^{5}$ Magnetobound states of positronium have also been proposed as an avenue for the synthesis of antihydrogen. ${ }^{6}$ A magnetobound state of positronium is a temporary correlated state of an electron and a positron, correlated due to the presence of an external magnetic field. ${ }^{7}$ A magnetobound state forms adiabatically as a result of a collision between the two particles, and the state persists while each particle experiences an $\boldsymbol{E} \times \boldsymbol{B}$ drift as a result of the electric field of the other particle. In schemes proposed in Ref. 6, magnetobound positronium drifts axially or radially relative to a magnetic field and combines with antiprotons to produce cold antihydrogen atoms. Magnetobound states of positronium could be produced for such a purpose within an electron-positron plasma. In the work presented here, cross-magnetic-field transport is studied in electron-positron plasmas under conditions where magnetobound positronium is expected to form.

Recent advances have been made to extend the understanding of collisional transport in magnetized plasmas affected by long-range collisions. ${ }^{8-10}$ Long-range collisions may play an important role in collisions with guiding-center impact parameters larger than the cyclotron radius and smaller than the Debye length. ${ }^{9}$ In this range, each particle can experience an $\boldsymbol{E} \times \boldsymbol{B}$ drift as a result of the electric field of the other particle. Enhanced diffusion by a factor of about 10 for cross-magnetic-field single-particle diffusion in a nonneutral ion plasma has been reported. ${ }^{9}$ A theoretical treatment reported in Ref. 10 included multiple collisions of the same pair of particles with guiding-center impact parameters larger than the cyclotron radius and smaller than the Debye length. The study found a factor of three enhancement due to multiple collisions involving the same two particles,

a) Author to whom correspondence should be addressed: cao@unt.edu combined with a factor of three enhancement due to longrange collisions. Also, a study has reported experimental confirmation of enhanced collisional drag due to long-range collisions in a magnetized non-neutral ion plasma. ${ }^{8}$

The present work evaluates a single-particle diffusion coefficient for independent binary collisions within an electron-positron plasma. For a collision involving an electron and a positron, the center of mass of the electron and positron can move across the magnetic field a distance several orders of magnitude larger than the size of the thermal cyclotron radius as a result of the formation of a magnetobound state of positronium. Figure 1 shows the trajectories of a positron and an electron which experience such a collision. The phenomenon represents an extreme type of transport associated with collision-based $\boldsymbol{E} \times \boldsymbol{B}$ drifts.

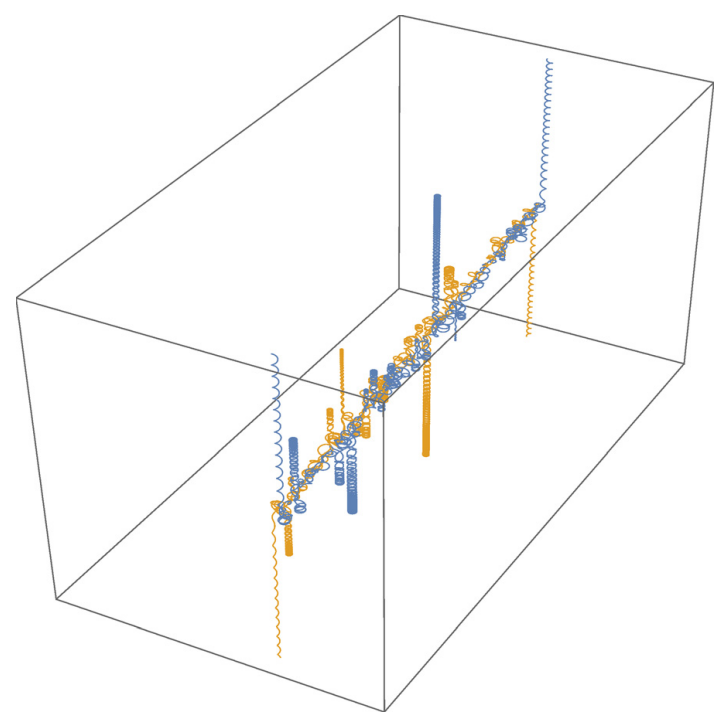

FIG. 1. Three dimensional view of the formation and dissociation of a magnetobound state of positronium. The two colors represent the separate trajectories of a positron and an electron. The two particles initially approach each other while following helical paths due to the presence of a magnetic field. The electron and positron drift across the magnetic field, while they remain spatially correlated with each other. 
The enhanced diffusion reported here due to collisionbased $\boldsymbol{E} \times \boldsymbol{B}$ drifts is likely to occur in mixing experiments for antihydrogen production within nested Penning traps, if antiprotons and some electrons (which may be leftover from the antiproton cooling process) are mixed with positrons. As is pointed out in Ref. 11, magnetically confined electronpositron plasmas may exhibit some remarkable stability properties at low densities. The present work indicates that such plasmas may also exhibit remarkable transport properties at low temperatures.

The cross-magnetic-field single-particle diffusion coefficient in electron-positron plasmas is evaluated using the classical trajectory Monte Carlo method. The motion of a positron and an electron is treated classically. A simulated binary collision considers their electric interaction under the influence of a uniform magnetic field. Coulomb's law accounts for the electric force exerted on particle $i$ by $j$ and is given by $\boldsymbol{F}_{\text {onibyj }}=k_{c} q_{i} q_{j} \boldsymbol{r}_{i j} / r_{i j}^{3}$, where $k_{c}$ is the Coulomb force constant, $q_{i}$ and $q_{j}$ are the charges, $r_{i j}=\left|\boldsymbol{r}_{i j}\right|$ is the relative distance, and $\boldsymbol{r}_{i j}=\boldsymbol{r}_{i}-\boldsymbol{r}_{j}$ is the vector position of particle $i$ relative to particle $j$. In SI units, $k_{c}=1 /\left(4 \pi \epsilon_{0}\right)$, where $\epsilon_{0}$ is the permittivity of free space. The force on particle $i$ by the magnetic field is $\boldsymbol{F}_{\text {oniby } B}=k_{L} q_{i} B\left(v_{y i} \hat{\boldsymbol{i}}-v_{x i} \hat{\boldsymbol{j}}\right)$, where $k_{L}$ is the Lorentz force constant ( $k_{L}=1$ in SI units), $\boldsymbol{B}(=B \hat{\boldsymbol{k}})$ is a uniform magnetic field parallel to the unit vector $\hat{\boldsymbol{k}},(\hat{\boldsymbol{i}}, \hat{\boldsymbol{j}}, \hat{\boldsymbol{k}})$ are the unit vectors of a Cartesian coordinate system, and $v_{x i}$, $v_{y i}, v_{z i}$ are the velocity components of particle $i$.

Newton's second law governs the classical motion of the particles. For particle $i, \boldsymbol{F}_{\text {onibyj }}+\boldsymbol{F}_{\text {oniby } B}=m_{i} \boldsymbol{a}_{i}$, where $m_{i}$ is the mass of particle $i$ and $\boldsymbol{a}_{i}$ is its acceleration. Therefore, the acceleration of particle $i$ is given by $k_{c} q_{i} q_{j} \boldsymbol{r}_{i j} /$ $r_{i j}^{3}+k_{L} q_{i} B\left(v_{y i} \hat{\boldsymbol{i}}-v_{x i} \hat{\boldsymbol{j}}\right)=m_{i} \boldsymbol{a}_{i}$. The velocity and position of each particle are functions of time. If the position is written as $\boldsymbol{r}_{i}(t)=x_{i}(t) \hat{\boldsymbol{i}}+y_{i}(t) \hat{\boldsymbol{j}}+z_{i}(t) \hat{\boldsymbol{k}}$ and the time derivative is written as $\boldsymbol{r}_{i}^{\prime}(t)=x_{i}^{\prime}(t) \hat{\boldsymbol{i}}+y_{i}^{\prime}(t) \hat{\boldsymbol{j}}+z_{i}^{\prime}(t) \hat{\boldsymbol{k}}$, then the equations of motion are

$$
\begin{aligned}
& \frac{k_{c} q_{i} q_{j}\left[x_{i}(t)-x_{j}(t)\right]}{\left(\left[x_{i}(t)-x_{j}(t)\right]^{2}+\left[y_{i}(t)-y_{j}(t)\right]^{2}+\left[z_{i}(t)-z_{j}(t)\right]^{2}\right)^{3 / 2}} \\
& \quad+k_{L} B q_{i} y_{i}^{\prime}(t)=m_{i} x_{i}^{\prime \prime}(t), \\
& \frac{k_{c} q_{i} q_{j}\left[y_{i}(t)-y_{j}(t)\right]}{\left(\left[x_{i}(t)-x_{j}(t)\right]^{2}+\left[y_{i}(t)-y_{j}(t)\right]^{2}+\left[z_{i}(t)-z_{j}(t)\right]^{2}\right)^{3 / 2}} \\
& -k_{L} B q_{i} x_{i}^{\prime}(t)=m_{i} y_{i}^{\prime \prime}(t), \\
& \quad k_{c} q_{i} q_{j}\left[z_{i}(t)-z_{j}(t)\right] \\
& \frac{\left(\left[x_{i}(t)-x_{j}(t)\right]^{2}+\left[y_{i}(t)-y_{j}(t)\right]^{2}+\left[z_{i}(t)-z_{j}(t)\right]^{2}\right)^{3 / 2}}{\quad=m_{i} z_{i}^{\prime \prime}(t) .}
\end{aligned}
$$

Here, $i=1, j=2$ applies for the positron and $i=2, j=1$ applies for the electron.

Classical trajectory Monte Carlo simulations are carried out by solving the equations of motion for each particle with sampled initial conditions. The electron and positron are considered (but not simulated) as having traveled oppositely from two positions separated infinite distances from the coordinate origin. At infinite separation, the particles are considered to reside within an electron-positron plasma and to be unaffected by each other, and the electric potential energy of the two-particle system is defined to be zero. The particles travel from infinite separation and are assumed to undergo independent cyclotron motion before the start of the simulation. The centers of their helical trajectories, their guiding centers, are treated as remaining unchanged until the start of the simulation. Henceforth, bar coordinates refer to guiding center coordinates, the subscript $(\infty)$ refers to when the particles are at infinite separation distance, the subscript (0) and the word "initial" refer to the start of the simulation, and the term "perpendicular" refers to perpendicular to the magnetic field.

The positron and electron plasmas are treated as being in thermal equilibrium. The distributions of particle velocities are assumed to follow a Maxwellian distribution for a given temperature $T$. This is implemented by sampling each of the three velocity components per particle $\left(v_{x i \infty}, v_{y i \infty}\right.$, $\left.v_{z i \infty}\right)$ as a random variate of a normal distribution with a zero value for the mean and a standard deviation equal to $\sqrt{k T / m_{i}}$. Here, $k$ is Boltzmann's constant.

The simulation starts at time $t=0$, when the two particles are at an axial distance of separation equal to $\zeta b$. Here, $b$ is the guiding center impact parameter, and a value of $\zeta=50$ is chosen to ensure that $b$ is much smaller than the initial axial separation distance. The (guiding center) impact parameter $b$ is taken to be the perpendicular distance between the initial guiding centers of the particles, each with a different gyroradius $\left(r_{c i}\right)$, prior to the start of the simulated motion (Fig. 2).

It is simpler to derive the sampling expression for the impact parameter $b$ with a coordinate system for which the initial guiding center of the positron coincides with the $z$

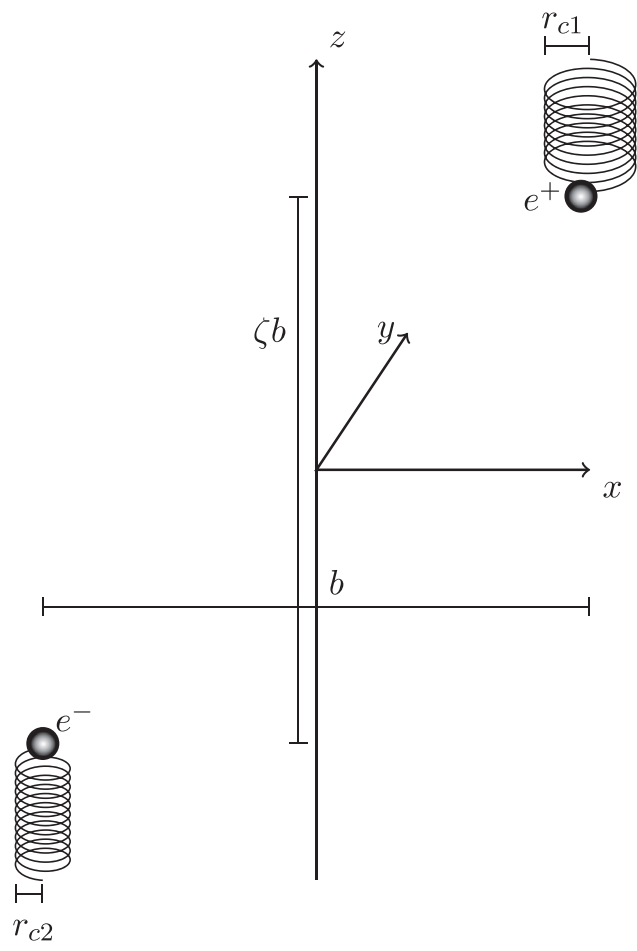

FIG. 2. Positron $e^{+}$and electron $e^{-}$at time $t=0$. (Not to scale.). 
axis. Afterward, the coordinate system is repositioned such that the two initial guiding centers are each at a radial distance $b / 2$ from the $z$ axis. The initial guiding center of the electron is equally likely to be located anywhere within a circle of radius $b_{\max }$, with the center of the circle coincident with the $z$ axis and the normal to the circle parallel to the $z$ axis. Here, $b_{\max }$ is a value of the impact parameter too large for magnetobound states to form. Let $b=\bar{r}_{20}=\sqrt{\bar{x}_{20}^{2}+\bar{y}_{20}^{2}}$ be an initial radial distance from the electron guiding center to the $z$ axis. The probability of lying within a radius less than $\bar{r}$ is equal to $P(b \leq \bar{r})=\pi \bar{r}^{2} / \pi b_{\max }^{2}$. By taking the inverse, the radial distance of the guiding center can be sampled randomly as $b=b_{\max } \sqrt{R}$, where $R$ is a pseudorandom number equally likely to have any value between 0 and 1 .

The coordinate system is reoriented for every collision such that particles start at the initial time with their guiding centers lying on the $x-z$ plane as shown in Fig. 2. The coordinates of the initial guiding centers of the positron and the electron are $\left(\bar{x}_{10}, \bar{y}_{10}\right)=(b / 2,0)$ and $\left(\bar{x}_{20}, \bar{y}_{20}\right)=(-b / 2,0)$. The initial positions and velocities of the particles are generated using the following equations:

$$
\begin{gathered}
x_{i 0}= \pm \bar{x}_{0}+r_{c i} \cos \left(\Theta_{i}\right), \\
y_{i 0}=r_{c i} \sin \left(\Theta_{i}\right), \\
z_{i 0}= \pm \zeta b / 2, \\
v_{x i 0}= \pm v_{\perp i 0} \sin \left(\Theta_{i}\right), \\
v_{y i 0}=\mp v_{\perp i 0} \cos \left(\Theta_{i}\right),
\end{gathered}
$$

$$
v_{z i 0}=\mp v_{z 0} .
$$

Here, $\bar{x}_{0}=b_{\max } \sqrt{R} / 2, b=2 \bar{x}_{0}$, the upper sign is used for the positron, the lower sign is for the electron, and the angle $\Theta_{i}$ is sampled as a random phase equally likely to have any value between 0 and $2 \pi$.

For a sufficiently large axial separation, the electron and positron will undergo independent cyclotron motion, each with a different cyclotron radius determined by the perpendicular speed $v_{\perp i}$ associated with the motion perpendicular to the magnetic field. At $t=0$, the initial single-particle cyclotron radius is given by $r_{c i}=m_{i} v_{\perp i 0} /\left(k_{L}\left|q_{i}\right| B\right)$. Since it is assumed that no change occurs in the perpendicular speed when the particles travel from the infinite separation distance to a distance at which $t=0$, the cyclotron radius and the perpendicular speed are constant during that motion, i.e., $r_{c i}$ $=r_{c i \infty}$, and $v_{\perp i 0}=\sqrt{v_{x i 0}^{2}+v_{y i 0}^{2}}=\sqrt{v_{x i \infty}^{2}+v_{y i \infty}^{2}}=v_{\perp i \infty}$.

When the particles are separated by a finite distance at the start of a simulation, the conservation of energy requires

$$
\begin{aligned}
\frac{1}{2} m_{1} & \left(v_{x 1 \infty}^{2}+v_{y 1 \infty}^{2}+v_{z 1 \infty}^{2}\right)+\frac{1}{2} m_{2}\left(v_{x 2 \infty}^{2}+v_{y 2 \infty}^{2}+v_{z 2 \infty}^{2}\right) \\
= & \frac{1}{2} m_{1}\left(v_{x 10}^{2}+v_{y 10}^{2}+v_{z 10}^{2}\right)+\frac{1}{2} m_{2}\left(v_{x 20}^{2}+v_{y 20}^{2}+v_{z 20}^{2}\right) \\
& +\frac{k_{c} q_{1} q_{2}}{r_{0}}
\end{aligned}
$$

Here, $v_{x i \infty}, v_{y i \infty}$, and $v_{z i \infty}$ are the components of the velocity of a particle at infinite separation, $v_{x i 0}, v_{y i 0}$, are $v_{z i 0}$ are the components of the initial velocity of a particle, and

$$
r_{0}=\sqrt{\left[2 \bar{x}_{0}+r_{c 1} \cos \left(\Theta_{1}\right)-r_{c 2} \cos \left(\Theta_{2}\right)\right]^{2}+\left[r_{c 1} \sin \left(\Theta_{1}\right)-r_{c 2} \sin \left(\Theta_{2}\right)\right]^{2}+4 \zeta^{2} \bar{x}_{0}^{2}}
$$

is the initial distance of separation between the two particles. Equation (10) accounts for the increase in kinetic energy experienced by each particle prior to $t=0$. (If the collision of two positrons is considered, then the kinetic energy of each particle would decrease.) In order to find the associated change in the axial velocity components using Eq. (10), an assumption is made regarding the frame of reference in which the collision occurs. For the present study, only crossmagnetic-field transport is of interest. Therefore, it is possible to choose a coordinate frame of reference for each collision such that the positron and the electron have the same initial axial speed, i.e., $\left|v_{z 10}\right|=\left|v_{z 20}\right|=v_{z 0}$. Altogether, the following equalities are used to simplify Eq. (10): $v_{z 20}=$ $-v_{z 10}=v_{z 0}, v_{x i 0}^{2}+v_{y i 0}^{2}=v_{x i \infty}^{2}+v_{y i \infty}^{2}$ and $m_{1}=m_{2}=m$. The magnitude of the axial velocity of each particle at the start of the simulation is then

$$
v_{z 0}=\sqrt{\frac{1}{2}\left(v_{z 1 \infty}^{2}+v_{z 2 \infty}^{2}\right)-\frac{k_{c} q_{1} q_{2}}{m r_{0}}}
$$

Each simulation is run until a time $t_{\max }$, which is the first time when the axial separation of the particles becomes larger than their initial axial separation. Therefore, the defining condition for $t_{\max }$ is when the following inequality is first satisfied:

$$
\left|z_{1}\left(t_{\max }\right)-z_{2}\left(t_{\max }\right)\right|>\zeta b .
$$

To evaluate a cross-magnetic-field single-particle diffusion coefficient, each trajectory simulation outputs a cross-magnetic-field drift distance experienced by each particle. The coordinates of the guiding center of a particle are used to calculate the cross-field drift distance of the particle. The coordinates of the guiding center of each particles are obtained using the following transformation: $\left(\bar{x}_{i}, \bar{y}_{i}\right)=\left(x_{i} \pm v_{y i} / \omega, y_{i} \overline{+} v_{x i} / \omega\right)$. Here, the upper sign is used for the positron, the lower sign for the electron, and $\omega=k_{L}\left|q_{i}\right| B / m_{i}$ is the lepton gyrofrequency. Let $\Delta r$ denote the distance between the positions of the guiding center of a particle at the end of the simulation (at $t=t_{\max }$ ) and at the beginning (at $t=0$ ). For the positron, the cross-magnetic-field drift distance is

$$
\Delta r=\sqrt{\left[\bar{x}_{1}\left(t_{\max }\right)-\frac{b}{2}\right]^{2}+\left[\bar{y}_{1}\left(t_{\max }\right)\right]^{2}} .
$$


The value, $b_{\max }=14 r_{c t}$, was chosen, which is a value of the impact parameter that was found by trial and error to be too large for magnetobound states to form. The thermal cyclotron radius is defined here as $r_{c t}=\sqrt{2 m k T /\left(k_{L}^{2} q^{2} B^{2}\right)}$. For $B=1 \mathrm{~T}$ and $T=4 \mathrm{~K}$, the thermal cyclotron radius is $6.26 \times 10^{-8} \mathrm{~m}$ for the positrons and electrons.

The rate of single-particle transport across the magnetic field is characterized by a diffusion coefficient. Modeling the diffusion as a random walk process, the diffusion coefficient for positrons is evaluated as

$$
D_{1 \perp}=\frac{1}{2}\left\langle n_{2} \sigma_{\max } u_{\infty}(\Delta x)^{2}\right\rangle_{b, \phi, v_{1 \infty}, v_{2 \infty}} .
$$

$n_{2}$ is the electron density. $\sigma_{\max }$ is equal to $\pi b_{\max }^{2} \cdot u_{\infty}$ is the relative axial speed of the two particles in the plasma. $\phi$ is an azimuthal angle between the $x$ axes of two coordinate systems with coincident origins and aligned $z$ axes, with one coordinate system rotated from collision to collision such that particles start at the initial time with their guiding centers lying on the $x-z$ plane as shown in Fig. 2. \langle\rangle$_{b, \phi, v_{1 \infty}, v_{2 \infty}}$ represents averages over the impact parameter, azimuthal angle, and velocity distributions of the positrons and electrons. $\Delta x=\Delta r \cos (\phi)$ is the cross-magnetic-field drift distance in the $x$ dimension of the unrotated coordinate system. $\phi$ is equally likely to have any value between zero and $2 \pi$, and

$$
\left\langle(\Delta x)^{2}\right\rangle_{\phi}=\left\langle(\Delta r)^{2} \cos ^{2}(\phi)\right\rangle_{\phi}=(\Delta r)^{2}\left\langle\cos ^{2}(\phi)\right\rangle_{\phi}=\frac{1}{2}(\Delta r)^{2} .
$$

With this, Eq. (15) reduces to

$$
D_{1 \perp}=\frac{n_{2} \sigma_{\max }}{4}\left\langle u_{\infty}(\Delta r)^{2}\right\rangle_{b, v_{1 \infty}, v_{2 \infty}} .
$$

The ratio $D_{1 \perp} / n_{2}$ is computed as

$$
\frac{D_{1 \perp}}{n_{2}}=\frac{\sigma_{\max }}{4} \sum_{k=1}^{N} u_{\infty}\left(\boldsymbol{v}_{1 \infty, k}, \boldsymbol{v}_{2 \infty, k}\right)\left[\Delta r\left(b_{k}, \boldsymbol{v}_{1 \infty, k}, \boldsymbol{v}_{2 \infty, k}\right)\right]^{2} .
$$

Here, $N$ is the number of trajectories simulated, and $\boldsymbol{v}_{1 \infty, k}, \boldsymbol{v}_{2 \infty, k}$ are velocities of the positron and electron in the plasma for the $k$ th collision having guiding center impact parameter $b_{k}$. Approximately 50000 trajectories were simulated for each value of plasma temperature $T$ and magnetic field strength $B$ considered.

Simulation results are presented in Figs. 3-5. Plasma parameters similar to those in mixing experiments for antihydrogen production within nested Penning traps are considered.

Figure 3 shows the dependence on temperature of the ratio of the single-particle diffusion coefficients for electronpositron and positron-positron binary Coulomb collisions $\left(D_{p e} / D_{p p}\right)$ for a fixed magnetic field strength $(B=1 \mathrm{~T})$. For the temperature range considered, the ratio increases sharply as the temperature decreases. At $T=4 \mathrm{~K}$, the single-particle diffusion coefficient for electron-positron Coulomb collisions is found to be more than four orders of magnitude

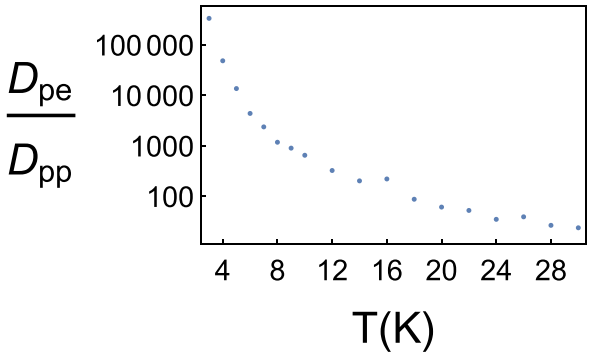

FIG. 3. Ratio of the single-particle diffusion coefficient for electron-positron collisions to the single-particle diffusion coefficient for positron-positron collisions as a function of temperature for a fixed magnetic field strength $(B=1 \mathrm{~T})$.

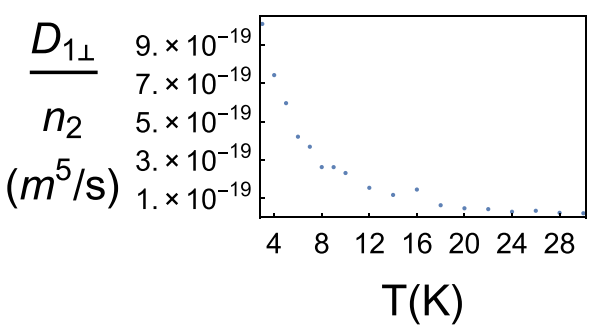

FIG. 4. The normalized single-particle diffusion coefficient associated with collisions between positrons and electrons within an electron-positron plasma as a function of temperature for a fixed magnetic field strength $(B=1 \mathrm{~T})$.

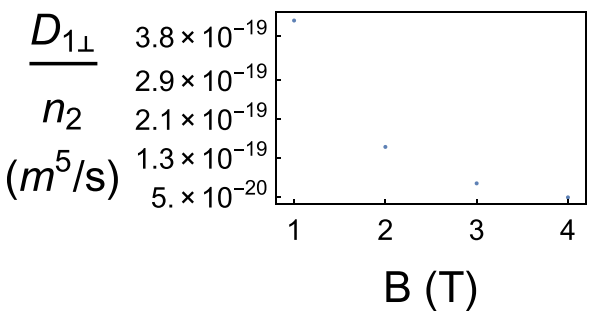

FIG. 5. The normalized single-particle diffusion coefficient associated with collisions between positrons and electrons within an electron-positron plasma as a function of magnetic field strength for a fixed temperature $(T=6 \mathrm{~K})$.

larger than the single-particle diffusion coefficient for positron-positron Coulomb collisions $\left(D_{p e} / D_{p p}>10^{4}\right.$ for $B=1 \mathrm{~T}$ and $T=4 \mathrm{~K}$ ). In contrast, collisional scattering processes associated with electron-positron Coulomb collisions are exactly the same as collisional scattering processes associated with positron-positron Coulomb collisions if the magnetic field is absent (i.e., if $B=0 \mathrm{~T}$ ). It was also found that the ratio $\left(D_{p e} / D_{p p}\right)$ drops below 10 for temperatures above $50 \mathrm{~K}$ when the magnetic field strength is $1 \mathrm{~T}$. The difference in the effect on the single-particle diffusion coefficient of these two types of collisions decreases as the temperature increases.

Figure 4 shows the normalized single-particle diffusion coefficient $D_{1 \perp} / n_{2}$ for electron-positron binary Coulomb collisions versus temperature for a fixed magnetic field strength $(B=1 \mathrm{~T})$. The normalized single-particle diffusion coefficient is found to increase by more than four orders of magnitude as the temperature decreases from $30 \mathrm{~K}$ to $4 \mathrm{~K}$, 
when the magnetic field strength is $1 \mathrm{~T}$. Figure 5 shows the normalized single-particle diffusion coefficient $D_{1 \perp} / n_{2}$ for electron-positron binary Coulomb collisions versus magnetic field strength for a fixed temperature $(T=6 \mathrm{~K})$. The normalized single-particle diffusion coefficient is found to increase as the magnetic field strength decreases from $4 \mathrm{~T}$ to $1 \mathrm{~T}$, with the temperature held fixed (at $T=6 \mathrm{~K}$ ).

In Ref. 7, counter-streaming electron and positron beams were considered for computing root-mean-square cross-magnetic-field drift distances of magnetobound positronium. The trends in Figs. 4 and 5 are consistent with prior results reported in Ref. 7, considering the very limited range of kinetic energies (3-6 K in temperature units) used in Ref. 7 and the normalization (by the cyclotron radius) that was used in Ref. 7.

The computations presented here are intended to apply for plasma conditions similar to those used for antihydrogen production, namely, $4 \mathrm{~K} \lesssim T \lesssim 30 \mathrm{~K}, n_{2} \approx 10^{13} \mathrm{~m}^{-3}$, and $B \approx 1 \mathrm{~T}$. An analytical theory that adequately describes cross-magnetic-field single-particle diffusion in electronpositron plasmas for such plasma conditions remains to be developed. It is noted that, for such conditions, a calculation of the conventional expression for the Coulomb logarithm yields a nonphysical $(\lessgtr 0)$ value. Therefore, a direct comparison is not done with the existing theory, such as that in Ref. 10 . It should also be noted that the transport process considered here is somewhat similar to antiproton transport that occurs by the production and ionization of antihydrogen during the mixing of antiprotons and positrons in a nested Penning trap environment. ${ }^{12}$

In the present work, the diffusion coefficient is defined using the mean-square step size of a random walk model and is not obtained from first principles. However, there exist different types of diffusion (e.g., sub-diffusion and superdiffusion defined in Refs. 13 and 14), which are characterized by the time dependence of the mean-square step size. For the simple random walk model used here, a particle takes a spatial step as a result of a binary collision that is considered to occur during a negligible amount of time, and each step is temporally separated from the previous step by a time period, $\tau=1 /\left(n_{2} \sigma_{\max } u_{\infty}\right)$. The size of each spatial step is obtained from a classical trajectory simulation of a binary collision, but the motion of the same particle is not followed for more than one collision. A simulation, such as a molecular dynamics simulation, could, in principle, be used to follow the temporal evolution of an electron-positron plasma and to classify the type of diffusion that occurs at low temperatures, provided that the computation time is manageable.

Figure 5 indicates that for an electron-positron plasma in a magnetic field of $1 \mathrm{~T}$ at a temperature of $6 \mathrm{~K}$ and with each species having a density of $10^{14} \mathrm{~m}^{-3}$, which is typical of the positron plasma density in antihydrogen production experiments, the single-particle diffusion coefficient is about
$4 \times 10^{-5} \mathrm{~m}^{2} / \mathrm{s}$. In comparison, the Bohm diffusion coefficient for the same plasma is $3 \times 10^{-5} \mathrm{~m}^{2} / \mathrm{s}$. However, the diffusion coefficient for high temperature plasmas, such as fusion plasmas, can be much larger.

In conclusion, simulation results indicate that the formation and dissociation of magnetobound states can significantly enhance cross-magnetic-field single-particle diffusion due to binary collisions in low temperature electron-positron plasmas. The phenomenon described here represents an extreme type of transport associated with collision-based $\boldsymbol{E} \times \boldsymbol{B}$ drifts, whereby a spatial correlation between two particles occurs while each particle experiences an $\boldsymbol{E} \times \boldsymbol{B}$ drift due to the electric field of the other particle. It was found that the cross-magnetic-field single-particle diffusion coefficient associated with electron-positron collisions can be more than four orders of magnitude larger than the crossmagnetic-field single-particle diffusion coefficient associated with positron-positron collisions under the same conditions. The formation of magnetobound states of positronium might serve as an intermediate step in a three-body recombination process that produces antihydrogen or positronium atoms.

Computational resources were provided by UNT's High Performance Computing Services. This material is based upon work supported by the National Science Foundation under Grant No. PHY-1500427 and by the Department of Energy under Grant No. DE-FG02-06ER54883.

${ }^{1}$ C. Amole, M. D. Ashkezari, M. Baquero-Ruiz, W. Bertsche, E. Butler, A. Capra, C. L. Cesar, M. Charlton, A. Deller, S. Eriksson, J. Fajans, T. Friesen, M. C. Fujiwara, D. R. Gill, A. Gutierrez, J. S. Hangst, W. N. Hardy, M. E. Hayden, C. A. Isaac, S. Jonsell, L. Kurchaninov, A. Little, N. Madsen, J. T. K. McKenna, S. Menary, S. C. Napoli, K. Olchanski, A. Olin, P. Pusa, C. Rasmussen, F. Robicheaux, E. Sarid, C. R. Shields, D. M. Silveira, C. So, S. Stracka, R. I. Thompson, D. P. van der Werf, J. S. Wurtele, A. Zhmoginov, and L. Friedland, Phys. Plasmas 20, 043510 (2013).

${ }^{2}$ C. A. Ordonez and D. L. Weathers, Phys. Plasmas 15, 083504 (2008).

${ }^{3}$ J. L. Pacheco, C. A. Ordonez, and D. L. Weathers, Phys. Plasmas 19, 102510 (2012).

${ }^{4}$ R. A. Lane and C. A. Ordonez, J. Phys. B: At., Mol. Opt. Phys. 49, 074008 (2016).

${ }^{5}$ A. S. Kadyrov, C. M. Rawlins, A. T. Stelbovics, I. Bray, and M. Charlton, Phys. Rev. Lett. 114, 183201 (2015).

${ }^{6}$ J. R. Correa and C. A. Ordonez, Phys. Plasmas (1994-present) 21, 082115 (2014).

${ }^{7}$ F. F. Aguirre and C. A. Ordonez, Phys. Rev. E 91, 033103 (2015).

${ }^{8}$ M. Affolter, F. Anderegg, D. H. E. Dubin, and C. F. Driscoll, Phys. Rev. Lett. 117, 155001 (2016).

${ }^{9}$ F. Anderegg, X.-P. Huang, E. M. Hollmann, C. F. Driscoll, T. M. O’Neil, and D. H. E. Dubin, Phys. Plasmas 4, 1552 (1997).

${ }^{10}$ D. H. E. Dubin, Phys. Rev. Lett. 79, 2678 (1997).

${ }^{11}$ P. Helander, Phys. Rev. Lett. 113, 135003 (2014).

${ }^{12}$ S. Jonsell, M. Charlton, and D. van der Werf, J. Phys. B: At., Mol. Opt. Phys. 49, 134004 (2016)

${ }^{13}$ R. Metzler and J. Klafter, Phys. Rep. 339, 1 (2000).

${ }^{14}$ G. M. Zaslavsky, Phys. Rep. 371, 461 (2002). 